\title{
Gender and Power Metrics Database: A living database of scales that have been used to measure constructs of gender, agency, power, and control
}

Evidence Project

Follow this and additional works at: https://knowledgecommons.popcouncil.org/departments_sbsr-rh How does access to this work benefit you? Let us know!

\section{Recommended Citation}

Evidence Project. 2020. "Gender and Power Metrics Database: A living database of scales that have been used to measure constructs of gender, agency, power, and control." Washington, DC: Population Council, The Evidence Project. 


\title{
Gender and Power Metrics Database
}

A living database of scales that have

been used to measure constructs of

gender, agency, power, and control.

\author{
.
}

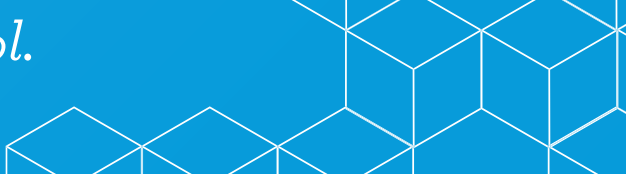

\section{TOOL}

The Evidence Project created a searchable database of gender- and power-related scales used in social, health, and behavioral science research. Indicators in the database have been used in in family planning (FP), reproductive health $(\mathrm{RH})$, intimate partner violence (IPV), and HIV research.

\section{HOW WE IDENTIFIED MEASURES}

Measures were identified using key term searches of papers in five electronic databases: Pubmed, EconLit, SocIndex, POPLine and Women's Studies International. Electronic searches were supplemented by hand searches of specific journals (e.g., Gender and Development; Studies in Family Planning; Culture, Health and Sexuality) and conference paper repositories. Eligible papers were published between 2000 and 2015 in English, included participants who were adolescents or of reproductive age (broadly defined as ages 10 to 49), and included psychometric information on scale properties related to a gender attitude/norm, agency or power indicator, and/or measured an association between a gender/agency/empowerment indicator and condom use, modern contraceptive use, IPV perpetration or victimization, or an HIV/STI outcome. Papers were excluded if they were unpublished, presented qualitative results only, or had a sample comprised of LGBT participants exclusively. The initial search identified over 19,000 citations, of which 2,130 papers were fully screened. The final sample included 668 papers that included 1,323 measures. Excluding duplicate measures, more than 600 unique measures remained.

\section{WHAT'S IN THE DATABASE}

The Gender and Power Metrics Database houses over 600 unique measures, many tested in multiple settings. It includes multi-item scales and single-item questions that reflect gender norms, personal views or beliefs about gender roles and norms, related feelings or emo-
Indicator catalog: distribution of scales by type (\%)

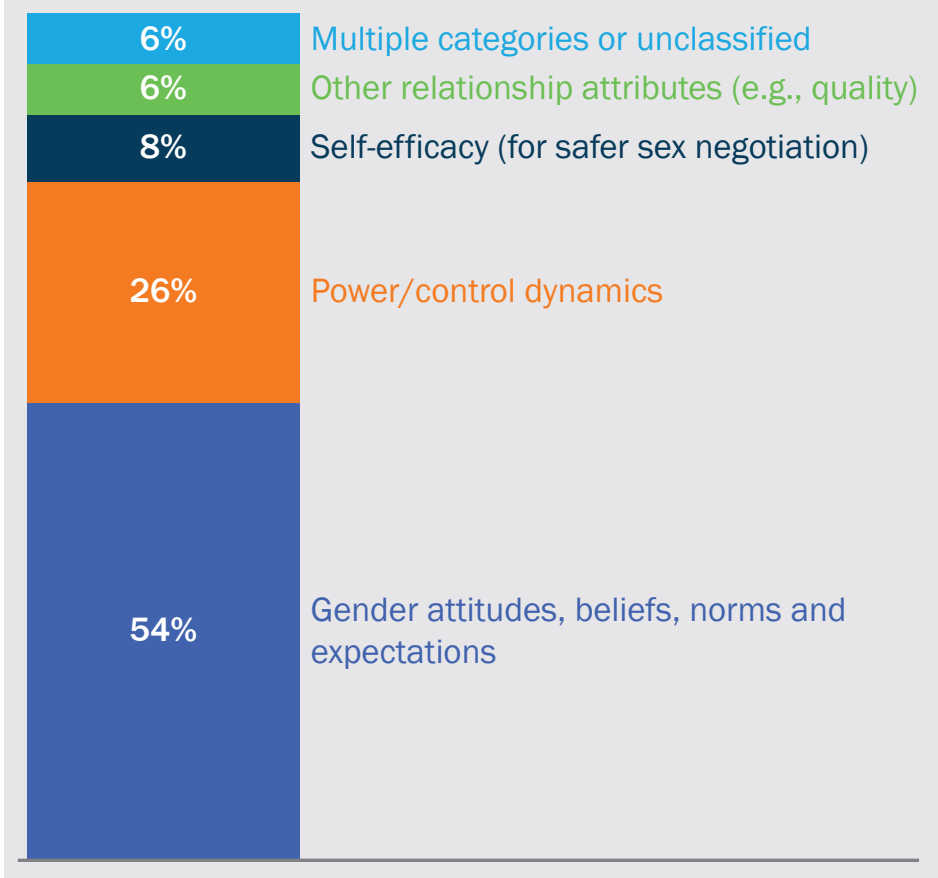

tions, gender role stress, gendered-dynamics, power and control in relationships, and individual-level agency and self-efficacy, among others.

The database provides information on the settings and populations where gender and power measures were implemented, citations for the papers, as well as extracted information on scale psychometric properties and, where available, the questions that comprise the scale. In cases where gender or power measures were measured in association with FP or IPV outcomes, the database provides additional information regarding the quantitative association between constructs.

The scale used most frequently was the Sexual Relationship Power Scale. ${ }^{1}$ Questions from the Demographic and Health Surveys' decision-making power module ${ }^{2}$ and items assessing attitudes toward IPV are also among the most frequently occurring. 


\section{HOW TO USE THE DATABASE}

The database is open access. It allows filtering and sorting to identify:

Measures currently in use and the broad category of scale (e.g., those related to gender norms, individual views or beliefs about gender roles/norms, gender-related power dynamics (in relationships or otherwise), self-efficacy for safer sex negotiation, or other aspects of relationships indicative of gendered power dynamics (relationship quality, trust, level of intimacy, etc.).

Geographic locations and populations where specific scales have been implemented.

The reliability and validity of scales (where applicable) used both within and across settings/populations.

Measures that have been analyzed against FP, IPV, RH, and HIV outcomes.

Search results can be exported as Excel or CSV files.
Researchers used the database to conduct a review of which measures* of gender inequitable norms, views, relations and practices are currently being used in the field, and which are most closely tied to male IPV perpetration.

* McCarthy, K. J., R. Mehta, and N. A. Haberland. 2018. "Gender, power, and violence: A systematic review of measures and their association with male perpetration of IPV," PLOS ONE 13(11): e0207091. doi: 10.1371/journal.pone.0207091.

Additionally, for those studies that have made some or all the questions that comprise their scales available, that information can be viewed by clicking "full" or "partial" in the Availability column.

${ }^{1}$ Pulerwitz, J., S. L. Gortmaker, and W. DeJong. 2000. "Measuring sexual relationship power in HIV/STD research." Sex Roles 42, 637-660. doil: 10.1023/A:1007051506972

${ }^{2}$ The DHS Program. 2020. "DHS overview." https://dhsprogram. com/what-we-do/survey-Types/dHs.cfm. Accessed 30 March 2020.

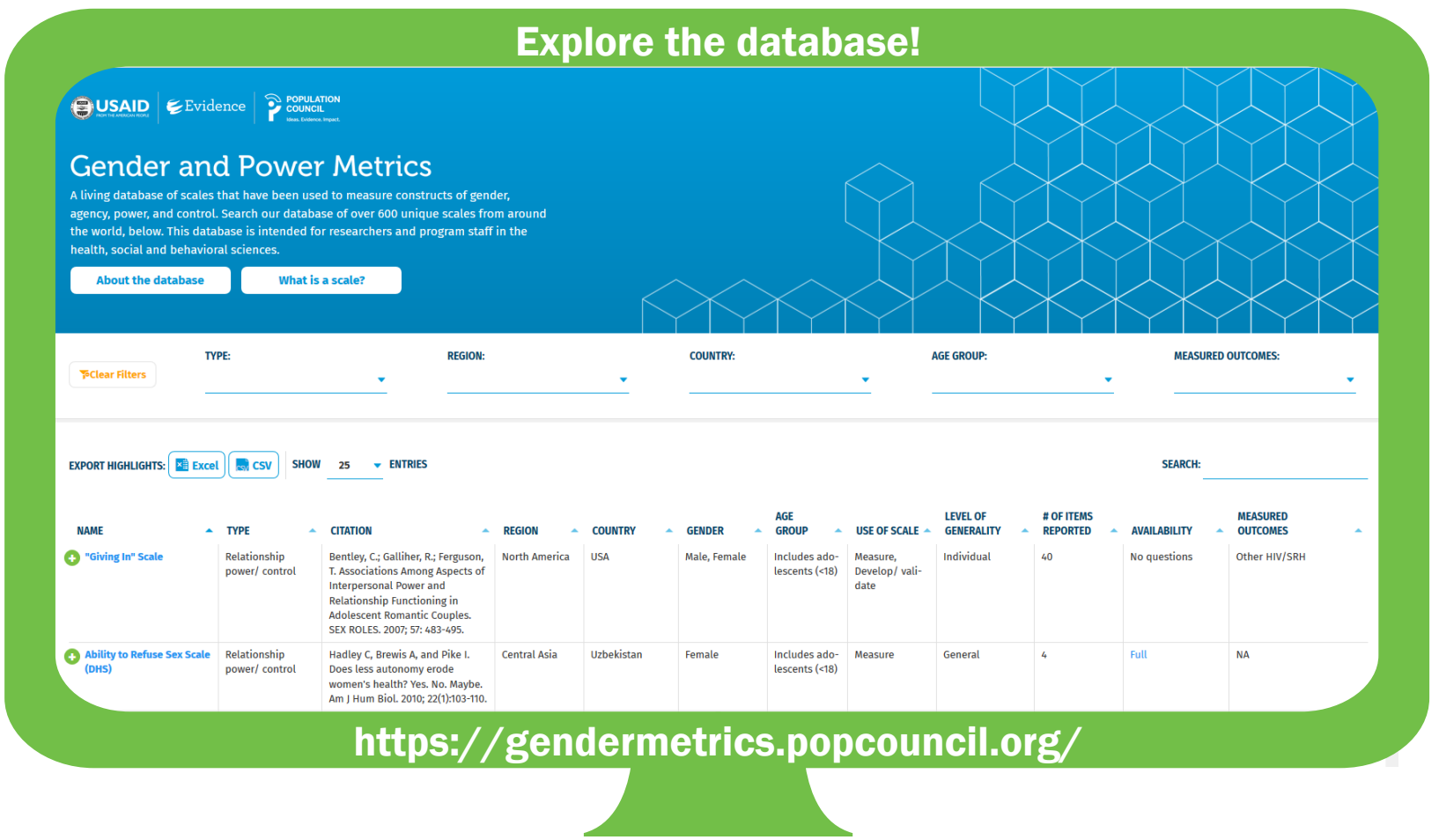

May 2020

USAID

Evidence
The Evidence Project uses implementation science-the strategic generation, translation, and use of evidence-to strengthen and scale up family planning and reproductive health programs to reduce unintended pregnancies worldwide. The Evidence Project is led by the Population Council.

(c) 2020. The Population Council, Inc.
The Evidence Project is made possible by the generous support of the American people through the United States Agency for International Development (USAID) under the terms of cooperative agreement no. AID-OAA-A-13-00087. The contents of this document are the sole responsibility of the Evidence Project and Population Council and do not necessarily reflect the views of USAID or the United States Government.

For more information, go to evidenceproject.popcouncil.org or send an e-mail to evidenceproject@popcouncil.org 